\title{
Recapitulating Amyloid $\beta$ and Tau Pathology in Human Neural Cell Culture Models-Clinical Implications
}

\author{
Se Hoon Choi, PhD, ${ }^{1}$ Young Hye Kim, PhD, ${ }^{2}$ Carla D'Avanzo, PhD, ${ }^{3}$ Jenna Aronson, BS, ${ }^{4}$ Rudolph E Tanzi, PhD ${ }^{5}$ and Doo Yeon Kim, PhD ${ }^{6}$ \\ 1. Instructor; Genetics and Aging Research Unit, MassGeneral Institute for Neurodegenerative Disease, Massachusetts General Hospital, Harvard Medical \\ School, Charlestown, Massachusetts, US 2. Senior Researcher, Biomedical Omics Group, Korea Basic Science Institute, Cheongju-si, Chungbuk, Republic of Korea. \\ 3. Postdoc Associate; 4. Research Technician; 4. Professor; 6. Assistant Professor, Genetics and Aging Research Unit, MassGeneral Institute for Neurodegenerative \\ Disease, Massachusetts General Hospital, Harvard Medical School, Charlestown, Massachusetts, us
}

\begin{abstract}
The "amyloid $\beta$ hypothesis" of Alzheimer's disease (AD) has been the reigning hypothesis explaining pathogenic mechanisms of AD over the last two decades. However, this hypothesis has not been fully validated in animal models, and several major unresolved factors remain. We recently developed a human neural cell culture model of $A D$ based on a three-dimensional (3D) cell culture system. This unique, cellular model recapitulates major events of the AD pathogenic cascade, including $\beta$-amyloid plaques and neurofibrillary tangles. Our 3D human neural cell culture model system provides a premise for a new generation of cellular AD models that can serve as a novel platform for studying pathogenic mechanisms and for high-throughput drug screening in a human brain-like environment.
\end{abstract}

\section{Keywords}

Alzheimer's disease, amyloid $\beta, A \beta, \beta$-amyloid precursor protein, APP, human neural progenitor cells, induced pluripotent stem cells, iPSCs, 3D culture model

\begin{abstract}
Disclosure: Se Hoon Choi, Young Hye Kim, Carla D'Avanzo, Jenna Aronson, Rudolph E Tanzi, and Doo Yeon Kim have no conflicts of interest to declare. No funding was received for the publication of this article.

Acknowledgements: This work is supported by grants from the Cure Alzheimer's fund to Se Hoon Choi, Rudolph E Tanzi, and Doo Yeon Kim; the Bio \& Medical Technology Development Program of the National Research Foundation (funded by the Korean government, MSIP [2015M3A9C7030151], Young Hye Kim); and National Institutes of Health (1RF1AG048080-01, Rudolph E Tanzi and Doo Yeon Kim; 5P01AG15379, Rudolph E Tanzi, and Doo Yeon Kim; 2R01AG014713, Doo Yeon Kim; 5R37MH060009, Rudolph E Tanzi). We would also like to thank Jae-Woong Ko and Kyu-Bong Han (Tech up cp., Ltd) for figure image illustrations and Enjana Bylykbashi (MGH) for critically reading the manuscript. Open Access: This article is published under the Creative Commons Attribution Noncommercial License, which permits any noncommercial use, distribution, adaptation, and reproduction provided the original author(s) and source are given appropriate credit.

Received: August 18, 2015 Accepted: September 18, 2015 Citation: US Neurology, 2015;11(2):102-5 DOl: http://doi.org/10.17925/USN.2015.11.02.102

Correspondence: Doo Yeon Kim, PhD, Genetics and Aging Research Unit, MassGeneral Institute for Neurodegenerative Disease, Massachusetts General Hospital, Harvard

Medical School, Charlestown, Massachusetts 02129, US. E: dkim@helix.mgh.harvard.edu
\end{abstract}

Alzheimer's disease (AD) is the most common neurodegenerative disease, clinically characterized by progressive memory loss. To date, an estimated 5.2 million people have the disease in the US, and the total number of people with $\mathrm{AD}$-related dementia is projected to rise to 13.8 million by $2050 .^{1,2}$ At present, there is no cure for the disease, and early clinical diagnosis is not yet available for the majority of patients.

The two main pathologic hallmarks of $A D$ are senile plaques (amyloid plaques) and neurofibrillary tangles (NFTs), which develop in brain regions responsible for memory and cognitive functions (i.e. cerebral cortex and limbic system). ${ }^{3}$ Senile plaques are extracellular deposits of amyloid- $\beta$ (A $\beta$ ) peptides, while NFTs are intracellular, filamentous aggregates of hyperphosphorylated tau protein. ${ }^{4}$

The identification of $A \beta$ as the main component of senile plaques by Doctors Glenner and Wong in $1984^{5}$ resulted in the original formation of the "amyloid hypothesis." According to this hypothesis, which was later renamed the "amyloid- $\beta$ cascade hypothesis" by Doctors Hardy and Higgins, ${ }^{6}$ the accumulation of $A \beta$ is the initial pathologic trigger in the disease, subsequently leading to hyperphosphorylation of tau, causing NFTs and, ultimately, neuronal death and dementia., ${ }^{4,7-10}$ Although the details have been modified to reflect new findings, the core elements of this hypothesis remain unchanged: excess accumulation of the pathogenic forms of $A \beta$, by altered $A \beta$ production and/or clearance, triggers the vicious pathogenic cascades that eventually lead to NFTs and neuronal death.

\section{The Amyloid $\beta$ Hypothesis-A Causal Link Between Amyloid $\beta$ and Neurofibrillary Tangles?} Over the last two decades, the A $\beta$ hypothesis of AD has reigned, providing the foundation for numerous basic studies and clinical trials. $4,7,10,11$ According to this hypothesis, the accumulation of $A \beta$, either 
by altered $A \beta$ production and/or clearance, is the initial pathologic trigger in the disease. The excess accumulation of $A \beta$ then elicits a pathogenic cascade including synaptic deficits, altered neuronal activity, inflammation, oxidative stress, neuronal injury, hyperphosphorylation of tau causing NFTs and, ultimately, neuronal death and dementia.,7-70 However, the $A \beta$ hypothesis has not been fully validated and several major unresolved matters remain. ${ }^{12-14}$ Recent failures of human clinical trials have raised concerns about whether blocking toxic $A \beta$ accumulation is sufficient to stop and even reverse the progression of downstream AD pathologies. ${ }^{15-17}$

One of the major unresolved factors of the $A \beta$ hypothesis is to show a direct causal link between $A \beta$ and NFTs. ${ }^{12-14}$ Studies have demonstrated that treatments with various forms of soluble $A \beta$ oligomers induced synaptic deficits and neuronal injury, as well as hyperphosphorylation of tau proteins, in mouse and rat neurons, which could lead to NFTs and neurodegeneration in vivo. ${ }^{18-21}$ However, transgenic AD mouse models carrying single or multiple human familial $A D$ (FAD) mutations in amyloid precursor protein (APP) and/or presenilin 1 (PS1) do not develop NFTs or robust neurodegeneration as observed in human patients, despite robust $A \beta$ deposition. ${ }^{13,22,23}$ Double and triple transgenic mouse models, harboring both FAD and tau mutations linked with frontotemporal dementia (FTD), are the only rodent models to date displaying both amyloid plaques and NFTs. However, the NFT pathology in these models stems mainly from the overexpression of human tau as a result of the FTD, rather than the FAD mutations. ${ }^{24,25}$

Failure of attempts at full recapitulation of AD pathologies in mice might be also due to fundamental species-specific differences between mice and humans. Indeed, adult mice do not express the six human isoforms of tau proteins and endogenous mouse tau seems to interfere with aggregation of human tau proteins. ${ }^{26}$

\section{Testing Amyloid $\beta$ Cascade Hypothesis in Human Neuronal Models}

Recent reprogramming technology has provided a new model to test the amyloid hypothesis. Specifically, induced pluripotent stem cells (iPSCS) can be generated from the fibroblasts of $A D$ patients harboring single FAD mutation either in APP or PS1. ${ }^{27-34}$ These neurons showed significant increases in the ratio of pathogenic $A \beta 42$ to $A \beta 40$ compared with non- $A D$ control neurons. $4,27,28,31,35-37$ Human neurons with the APP duplication FAD mutation also displayed robust increases in total $A \beta$ levels due to heightened levels of APP, the precursor protein for A $\beta$ generation. ${ }^{27,33}$ Similarly, trisomy 21 (Down syndrome) neurons also showed robust increases in total A $\beta$ levels due to the APP gene duplication, which is located on chromosome $21 .{ }^{38}$

Human neurons carrying FAD mutations are an optimal model to test whether elevated levels of pathogenic $A \beta$ trigger pathogenic cascades including NFTs, since those cells truly share the same genetic background that induces FAD in humans. Indeed, Israel et al., observed elevated tau phosphorylation in neurons with an APP duplication FAD mutation. ${ }^{33}$ Blocking $A \beta$ generation by $\beta$-secretase inhibitors significantly decreased tau phosphorylation in the same model, but $\gamma$-secretase inhibitor, another A $\beta$ blocker, did not affect tau phosphorylation..$^{33}$ Neurons with the APP V717I FAD mutation also showed an increase in levels of phospho tau and total tau levels. ${ }^{28}$ More importantly, Muratore and colleagues showed that treatments with $A \beta$-neutralizing antibodies in those cells significantly reduced the elevated total and phospho tau levels at the early stages of differentiation, suggesting that blocking pathogenic $A \beta$ can reverse the abnormal tau accumulation in APP V717I neurons. ${ }^{28}$

Recently, Moore et al. also reported that neurons harboring the APP V717I or the APP duplication FAD mutation showed increases in both total and phospho tau levels. ${ }^{27}$ Interestingly, altered tau levels were not detected in human neurons carrying PS1 FAD mutations, which significantly increased pathogenic A 342 species in the same cells. ${ }^{27}$ Treatments with $\beta$-secretase inhibitor significantly decreased phospho and total tau levels in the APP V717I and the APP duplication models, but $\gamma$-secretase inhibitor could not reduce abnormal tau accumulation in the same cells. ${ }^{27}$ These data suggest that elevated tau levels in these models were not due to extracellular $A \beta$ accumulation but may possibly represent an early stage of tauopathy. Alternatively, it may result from developmental alterations induced by the APP FAD mutations. Further studies will be needed to clarify the pathogenic importance of tau changes in human iPSC-derived AD neurons.

One of the challenges of replicating tauopathy in human iPSC-derived neurons is that wild-type human iPSC-derived neurons, despite longer differentiation (>100 days), do not fully express adult tau splicing isoforms. ${ }^{39-41}$ The presence of select FTD tau mutations enhances the expression of adult 4-repeat tau splicing isoforms. ${ }^{39-41}$ While control wild-type neurons do not express adult tau isoforms in the same conditions. ${ }^{39-41}$ This clearly limits the recapitulation of human tauopathy, in which 4-repeat tau plays an important role, in human iPSC-derived neurons without FTD tau mutations.

As summarized, most human FAD neurons showed significant increases in pathogenic A $\beta$ species, while only APP FAD neurons showed altered tau metabolism possibly representing early stages of tauopathy. However, all of these human FAD neurons failed to recapitulate robust extracellular amyloid plaques, NFTs, or any signs of neuronal death, as predicted in the amyloid hypothesis. Difficulty proving the amyloid hypothesis thus far in FAD IPSC neurons might be a consequence of the low levels of pathogenic $A \beta$ in these cultures. Average $A \beta$ levels in brains of $A D$ patients are much higher than those achieved in FAD iPSC-derived neuronal cells. ${ }^{27-34,42}$ It is possible that human iPSC-derived FAD neurons may not be suitable for generation of elevated $A \beta$ levels on par with levels found in the brains of AD patients. ${ }^{43}$

\section{Modeling Amyloid Plaques and Neurofibrillary Tangles in a Human Neural 3D Culture System}

In our recent study, we moved one step closer to proving the amyloid hypothesis. By generating human neural stem cell lines carrying multiple mutations in APP as well as PS1, we achieved high levels of pathogenic A 342 comparable to those in brains of $A D$ patients. ${ }^{44-46}$ Co-expression of multiple FAD mutations in APP and PS1 has been previously employed for generations of $A D$ transgenic mouse models. This strategy has been shown to increase aggregation-prone $A \beta 42$ levels through both dramatic acceleration of onset and increased total levels of $A \beta$ deposition. .22,23,47 $^{2}$

Secreted $A \beta$ in a conventional 2D cell culture system was observed to diffuse into the cell culture media, and be removed during media changes, precluding any possibility of aggregation. This finding led us to adopt a novel 3D Matrigel ${ }^{\circledR}$ culture system to create an environment in which secreted $A \beta$ accumulates, accelerating $A \beta$ aggregation. ${ }^{44,45} \mathrm{After} 6$ weeks of differentiation 


\section{Figure 1: Platform for AD Drug Screening in Human Neural Progenitor Cells with FAD Mutations in a 3D Culture System, which Successfully Reproduce Human AD Pathogenesis (Amyloid Plaque-driven Tauopathy)}

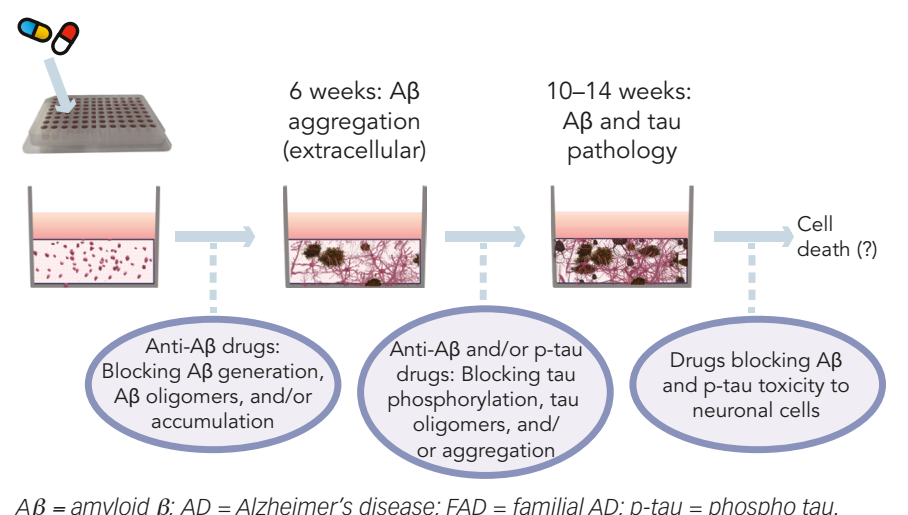

in our 3D Matrigel system, our FAD ReNcell VM human neural precursor cells showed robust extracellular $A \beta$ deposits and detergent sodium dodecyl sulfate (SDS)-resistant A $\beta$ aggregates ( $A \beta$ dimers, trimers, and tetramers). ${ }^{44,45}$ Importantly, we observed accumulations of hyperphosphorylated tau proteins in somatodendritic compartments, which were also present in detergent-insoluble fractions. ${ }^{44,45}$ Immunoelectron microscopy confirmed the presence of detergent-insoluble filamentous structures labeled by tau antibodies. ${ }^{44}$ Taken together, these observations clearly demonstrated the presence of $A \beta$ plaques and NFT-like pathologies in our 3D human $A D$ culture model. Notably, these AD pathologies were induced solely by FAD mutations without co-expressing human tau FTD mutations.

Next, we tested the direct causal link between excess accumulation of $A \beta$ and NFT-like pathology in our 3D human cellular AD culture system, as predicted in the amyloid hypothesis. Inhibition of $A \beta$ generation with either $\beta$ - or $\gamma$-secretase inhibitors decreased $A \beta$ deposits while also dramatically reducing phospho tau accumulation, particularly in dendrites and axons. ${ }^{44}$ Our results support the amyloid $\beta$ hypothesis, which posits that FAD mutations cause robust accumulation of $A \beta$, thus triggering hyperphosphorylated tau pathology.

Although our 3D human cellular $A D$ model was able to simulate major pathogenic events of $A D$, many challenges remain in comprehensive recapitulation. One major disadvantage of our model is manifested in the use of transgenic overexpression of APP and PS1 to generate elevated A $\beta$ levels. This nonphysiologic overexpression of APP may lead to supplementary pathogenic effects in addition to the accumulation of $A \beta$, as observed in transgenic $A D$ mouse models. ${ }^{48}$ Furthermore, the limitations in differentiating ReN cells into mature forebrain neurons, which are most affected in $A D$, may also pose a formidable challenge in fully reconstituting pathogenic cascades of $A D$.

\section{Clinical Implication of Human Cellular Models of Alzheimer's Disease}

One of the major advantages of human cellular models of $A D$ is that they can provide platforms for high-throughput screening (HTS) of new AD drugs in a human brain-like environment. Our studies clearly demonstrated the advantage of using human neural cell culture systems in recapitulating A $\beta$-induced tauopathy, which was not feasible in mouse models. ${ }^{44-46}$ Our 3D human cellular AD model can also provide a powerful platform for HTS of candidate $A D$ drugs that can reduce $A \beta$ and/or tau pathology in a single system, which is not possible in current AD models (see Figure 1). Also, our 3D culture systems allow scalable plating techniques, from thick-layer 6- and 24-well formats to a 96-well thin-layer design, which can fit largescale HTS or high-content screening (HCS). ${ }^{44-46}$ An additional strength of our immortalized and single-clonal human neural progenitor cells is their rapid proliferation and stability throughout repeated passages.

Another potential application of human cellular models of $A D$ is to validate/ optimize current therapeutic approaches for $A D$ in a human brain-like environment. For example, various antibodies against monomeric and/or oligomeric $A \beta$ species have been under human clinical trials, mostly based on success in AD mouse models. ${ }^{49}$ As shown by Muratore et al., the efficacy of $\mathrm{A} \beta$ neutralizing antibodies can also be tested in a human neural cell culture model of $A D .^{28}$ In addition to the impact on toxic $A \beta$ species, our $3 D$ culture model can test if these antibodies can block tau pathologies in $3 D$ human neural cell culture systems. ${ }^{44-46}$ Human cellular AD models can also be used to determine optimal doses of candidate AD drugs to block $A \beta$ and/or tau pathology without affecting neuronal survival (see Figure 1). Indeed, a recent study has shown that human iPSC-derived neurons are more resistant to nonsteroidal anti-inflammatory drug (NSAID)based $\boldsymbol{\gamma}$-secretase modulation compared with rodent neurons, which may explain the failure of human clinical trials of select NSAIDs. ${ }^{50}$

In addition to drug screening, human cellular AD models can be used to explore molecular mechanisms underlying AD pathogenesis, which could provide novel druggable targets to reduce AD pathology. ${ }^{51}$ While human iPSC-derived AD models allow study of pathogenic mechanisms under physiologic conditions, our 3D model provides insight after pathologic accumulation of $\beta$-amyloid and NFT formation, which are present in moderate to advanced AD.

While much progress has been made, many challenges still lie on the path to creating human neural cell culture models that comprehensively recapitulate pathogenic cascades of $A D$. A major difficulty lies in reconstituting the brain regions most affected in $A D$ : the hippocampus and specific cortical layers. Recent progress in 3D culture technology, such as "cerebral organoids" may also be helpful in rebuilding the brain structures that are affected by AD in a dish. ${ }^{52,53}$ These "cerebral organoids" were able to model various discrete brain regions including human cortical areas, ${ }^{52}$ allowing them to reproduce microcephaly, a brain developmental disorder. Similarly, pathogenic cascades of $A D$ may be recapitulated in cortex-like structures using this model. Adding neuroinflammatory components, such as microglial cells, which are critical in AD pathogenesis, will illuminate the validity of the amyloid $\beta$ hypothesis. Reconstitution of robust neuronal death stemming from $A \beta$ and tau pathologies will be the next major step in comprehensively recapitulating $A D$ in a cellular model.

Another major challenge lies in developing a precise model for sporadic AD. Currently all of the AD mouse models harbor FAD mutations, which are able to reconstitute pathogenic mechanisms of FAD. Conversely, sporadic $A D$ is caused by both genetic and environmental factors that have not been fully characterized yet. Human iPSC-derived neurons from sporadic AD patients 
can provide a model for sporadic AD. 32,33,54,55 Indeed, some human iPSC cell lines derived from sporadic $A D$ patients showed elevated levels of $A \beta .^{33,54}$ However, these $A \beta$ increases have not been consistently observed in other sporadic iPSC-derived neurons. ${ }^{32,33} \mathrm{Also}$, no severe AD pathologies, including extracellular aggregation of $\beta$-amyloid or robust tauopathy, were observed in these cells. $32,33,54,55$ These inconsistencies clearly demonstrate the technical difficulties of modeling sporadic AD even with human iPSCS derived from patients. However, according to the A $\beta$ hypothesis, sporadic $A D$ and FAD share common pathogenic pathways. Thus, drugs that can block $A \beta$ toxicity may work for both FAD and sporadic AD patients, which justifies the use of FAD models to develop drugs for sporadic AD patients.

We predict that further advances in human stem cell technology, as well as recent progress in $3 D$ culture technology, will generate cellular $A D$ models that can more precisely mimic $A D$ pathogenesis in a dish. These AD models will accelerate discovery of new AD drugs and also enable dissection of molecular mechanisms underlying the pathogenic cascades of $A D$.
1. 2015 Alzheimer's disease facts and figures, Alzheimers Dement, 2015;11:332-84

2. Hebert LE, Weuve J, Scherr PA, Evans DA, Alzheimer disease in the United States (2010-2050) estimated using the 2010 census, Neurology, 2013;80:1778-83.

3. Alzheimer A, Förstl H, Levy R, On certain peculiar diseases of old age, Hist Psychiatry, 1991;2(5 Pt 1):71-101.

4. Tanzi RE, Bertram L, Twenty years of the Alzheimer's disease amyloid hypothesis: a genetic perspective, Cell, 2005;120:545-55

5. Glenner GG, Wong CW, Alzheimer's disease: initial report of the purification and characterization of a novel cerebrovascular amyloid protein, Biochem Biophys Res Commun, 1984;120:885-90.

6. Hardy JA, Higgins GA, Alzheimer's disease: The amyloid cascade hypothesis, Science, 1992;256:184-5.

7. Hardy J, The amyloid hypothesis of Alzheimer's disease: Progress and problems on the road to therapeutics, science, 2002;297:353-6

8. Selkoe D, Alzheimer's disease is a synaptic failure, Science, 2002;298:789-91.

9. Benilova I, Karran E, De Strooper B, The toxic A $\beta$ oligomer and Alzheimer's disease: an emperor in need of clothes, Nat Neurosci, 2012:15:349-57.

10. Karran $E$, Mercken $M$, Strooper BD, The amyloid cascade hypothesis for Alzheimer's disease: an appraisal for the development of therapeutics, Nat Rev Drug Discov, 2011;10:698-712.

11. Tanzi RE, A brief history of Alzheimer's disease gene discovery J Alzheimers Dis, 2013;33:S5-13.

12. Morris GP, Clark IA, Vissel B, Inconsistencies and controversies surrounding the amyloid hypothesis of Alzheimer's disease, Acta Neuropathol Commun, 2014:2:135.

13. Armstrong RA, A critical analysis of the 'amyloid cascade hypothesis', Folia Neuropathol, 2014;52:211-25.

14. Herrup $K$, The case for rejecting the amyloid cascade hypothesis, Nat Neurosci, 2015;18:794-9.

15. De Strooper B, Lessons from a failed $\gamma$-secretase Alzheimer trial, Cell, 2014;159:721-6.

16. Doody RS, Thomas RG, Farlow M, et al., Phase 3 trials of solanezumab for mild-to-moderate Alzheimer's disease, N Eng/J Med, 2014:370:311-21.

17. Salloway S, Sperling R, Fox NC, et al., Two phase 3 trials of bapineuzumab in mild-to-moderate Alzheimer's Disease, N Engl J Med, 2014;370:322-33

18. Lambert MP, BarloW AK, Chromy BA, et al., Diffusible, nonfibrillar ligands derived from Abeta1-42 are potent central nervous system neurotoxins, Proc Natl Acad Sci U S A, 1998;95:6448-53.

19. Ferreira A, Lu Q, Orecchio L, Kosik KS, Selective phosphorylation of adult tau isoforms in mature hippocampal neurons exposed to fibrillar A beta, Mol Cell Neurosci, 1997:9:220-34.

20. Shankar GM, Bloodgood BL, Townsend M, et al., Natural oligomers of the Alzheimer amyloid-beta protein induce reversible synapse loss by modulating an NMDA-type glutamate receptor-dependent signaling pathway, J Neurosci, 2007;27:2866-75.
21. Jin M, Shepardson $N$, Yang T, et al., Soluble amyloid-protein dimers isolated from Alzheimer cortex directly induce Tau hyperphosphorylation and neuritic degeneration, Proc Natl Acad Sci U S A, 2011;108:5819-24.

22. Götz J, Ittner LM, Animal models of Alzheimer's disease and frontotemporal dementia, Nat Rev Neurosci, 2008:9:532-44.

23. Chin J, Selecting a mouse model of Alzheimer's disease, Methods Mol Biol, 2011;670:169-89.

24. Lewis J, Dickson DW, Lin WL, et al., Enhanced neurofibrillary degeneration in transgenic mice expressing mutant tau and APP, Science, 2001;293:1487-91

25. Oddo S, Caccamo A, Shepherd JD, et al., Triple-transgenic mode of Alzheimer's disease with plaques and tangles: intracellular Abeta and synaptic dysfunction, Neuron, 2003;39:409-21.

26. Ando K, Leroy K, Héraud C, et al., Accelerated human mutan tau aggregation by knocking out murine tau in a transgenic mouse model, Am I Pathol, 2011:178:803-16.

27. Moore S, Evans LD, Andersson T, et al., APP metabolism regulates tau proteostasis in human cerebral cortex neurons, Cell Reports, 2015;11:689-96.

28. Muratore CR, Rice HC, Srikanth P, et al., The familial Alzheimer's disease APPV717I mutation alters APP processing and Tau expression in IPSC-derived neurons, Hum Mol Genet, 2014:23:3523-36

29. Mohamet L, Familial Alzheimer's disease modelling using induced pluripotent stem cell technology, WJSC, 2014;6:239.

30. Duan L, Bhattacharyya BJ, Belmadani A, et al., Stem cell derived basal forebrain cholinergic neurons from Alzheimer's disease patients are more susceptible to cell death, Mol Neurodegener 2014;9:3

31. Sproul AA, Jacob S, Pre ED, et al., Characterization and molecular profiling of PSEN1 familial Alzheimer's disease IPSCderived neural progenitors, PLOS ONE, 2014'9:e84547.

32. Kondo T, Asai M, Tsukita K, et al., Modeling Alzheimer's disease with iPSCs reveals stress phenotypes associated with intracellular $A \beta$ and differential drug responsiveness, Cell Stem Cell, 2013;12:487-96.

33. Israel MA, Yuan SH, Bardy C, et al., Probing sporadic and familia Alzheimer's disease using induced pluripotent stem cells, Nature, 2012:482:216-20.

34. Yagi $T$, Ito D, Okada Y, et al., Modeling familial Alzheimer's disease with induced pluripotent stem cells, Hum Mol Genet, 2011:20:4530-9

35. Woodruff G, Young JE, Martinez FJ, et al., The presenilin-1 $\Delta E$ mutation results in reduced $\gamma$-secretase activity, but not tota loss of PS1 function, in isogenic human stem cells, Cell Rep, 2013:5:974-85.

36. Koch P, Tamboli IY, Mertens I, et al., Presenilin-1 L166P mutant human pluripotent stem cell-derived neurons exhibit partial loss of $\gamma$-secretase activity in endogenous amyloid- $\beta$ generation, Am J Pathol, 2012;180:2404-16.

37. Hu W, Qiu B, Guan W, et al., Direct conversion of normal and Alzheimer's disease human fibroblasts into neuronal cells by small molecules, Cell Stem Cell, 2015;17:204-12.

38. Shi Y, Kirwan P, Smith J, et al., A human stem cell model of early
Alzheimer's disease pathology in Down syndrome, Sci Transl Med, 2012;4:124ra29-124ra29

39. Sposito T, Preza E, Mahoney CJ, et al., Developmental regulation of tau splicing is disrupted in stem cell-derived neurons from frontotemporal dementia patients with the $10+16$ splice-site mutation in MAPT, Hum Mol Genet, 2015:24:5260-9.

40. Iovino M, Agathou S, González-Rueda A, et al., Early maturation and distinct tau pathology in induced pluripotent stem cellderived neurons from patients with MAPT mutations, Brain, 2015 [Epub ahead of print].

41. Ehrlich M, Hallmann AL, Reinhardt P, et al., Distinct neurodegenerative changes in an induced pluripotent stem cell model of frontotemporal dementia linked to mutant tau protein, Stem Cell Reports, 2015;5:83-96.

42. Wang J, Dickson DW, Trojanowski JQ, Lee VM, The levels of soluble versus insoluble brain Abeta distinguish Alzheimer's disease from normal and pathologic aging, Exp Neurol, 1999;158:328-37.

43. Kim DY, Carey BW, Wang $\mathrm{H}$, et al., BACE1 regulates voltagegated sodium channels and neuronal activity, Nat Cell BiOl 2007;9:755-64.

44. Choi SH, Kim YH, Hebisch M, et al., A three-dimensional human neural cell culture model of Alzheimer's disease, Nature, 2014:515:274-8.

45. Kim YH, Choi SH, D'Avanzo C, et al., A 3D human neural cell culture system for modeling Alzheimer's disease, Nat Protoc, 2015;10:985-1006

46. D'Avanzo C, Aronson J, Kim YH, et al., Alzheimer's in 3D culture: Challenges and perspectives, Bioessays, 2015;37:1139-48.

47. Duff K, Rao MV, Progress in the modeling of neurodegenerative diseases in transgenic mice, Curr Opin Neurol, 2001:14:441-7.

48. Nilsson P, Saito T Saido TC, New mouse model of Alzheimer's, ACS Chem Neurosci, 2014:5:499-502.

49. Wisniewski T, Goñi F, Immunotherapeutic approaches for Alzheimer's disease, Neuron, 2015;85:1162-76.

50. Mertens J, Stüber K, Wunderlich P, et al., APP processing in human pluripotent stem cell-derived neurons is resistant to NSAID-based $\gamma$-secretase modulation, Stem Cell Reports, 2013:1:491-8

51. Livesey FJ, Human stem cell models of dementia, Hum Mol Genet, 2014:23:R35-R39.

52. Lancaster MA, Renner M, Martin CA, et al., Cerebral organoids model human brain development and microcephaly, Nature, 2013;501:373-9.

53. Panca AM, Sloan SA, Clarke LE, et al., Functional cortical neurons and astrocytes from human pluripotent stem cells in 3D culture, Nat Methods, 2015;12:671-8.

54. Duan L, Bhattacharyya BJ, Belmadani A, et al., Stem cell derived basal forebrain cholinergic neurons from Alzheimer's disease patients are more susceptible to cell death, Mol Neurodegener. 2014;9:3.

55. Hossini AM, Megges M, Prigione A, et al., Induced pluripotent stem cell-derived neuronal cells from a sporadic Alzheimer's disease donor as a model for investigating AD-associated gene regulatory networks, BMC Genomics, 2015;16:84. 